\title{
Risk factors for the development of respiratory distress syndrome and transient tachypnoea in newborn infants
}

\author{
C. Dani*, M.F. Reali*, G. Bertini*, L. Wiechmann*, A. Spagnolo**, M. Tangucci**, F.F. Rubaltelli*, \\ and the Italian Group of Neonatal Pneumology
}

Risk factors for the development of respiratory distress syndrome and transient tachypnoea in newborn infants. C. Dani, M.F. Reali, G. Bertini, L. Wiechmann, A. Spagnolo, M. Tangucci, F.F. Rubaltelli, and the Italian Group of Neonatal Pneumology. (C) ERS Journals Ltd 1999.

ABSTRACT: Respiratory distress syndrome (RDS) and transient tachypnoea (TT) are the most frequent acute respiratory diseases in the newborn. This study investigated the risk factors for RDS and TT in newborn infants.

A population of 63,537 newborns was enrolled in a 12-month survey in Italy, 734 $(1.15 \%)$ affected by RDS and $594(0.93 \%)$ affected by TT. Multivariate regression analysis of maternal and perinatal data and the calculation of odds ratios (with $95 \%$ confidence intervals) were performed.

It was demonstrated that gestational age, birthweight, maternal age, elective and emergency caesarean section (CS), and male sex were risk factors for RDS, while gestational age, maternal diseases, twinning, birthweight, operative vaginal delivery, elective and emergency CS, and male sex were risk factors for TT.

The data confirm previous reports and demonstrate that advanced maternal age is a risk factor for RDS, while ruling out maternal diseases as independent risk factors for RDS.

Eur Respir J 1999; 14: 155-159.

Morbidity and mortality in the neonatal period are mainly due to respiratory disorders, respiratory distress syndrome (RDS), with transient tachypnoea (TT) being the most frequent. Recently, only a few area-based prospective studies have been carried out evaluating the occurrence of acute respiratory illness and its risk factors. Moreover, virtually none have been performed after the introduction of antenatal induction of lung maturity. In 1981, HJALMARSON [1] related the frequency of acute respiratory disorders to gestational age, birthweight, sex and postnatal asphyxia and, in 1994, Bonafè and Rubaltelli [2] related the incidence of neonatal respiratory disorders to gestational age and birthweight.

The purpose of this study was to investigate maternal and perinatal risk factors for RDS and TT in newborn infants. The Italian Group of Neonatal Pneumology performed a multicentre prospective study with the primary end point of evaluation of the incidence of major acute neonatal respiratory illnesses and related complications [3]. The collected data set was large enough to permit examination of the perinatal and maternal risk factors for the development of RDS and of TT in the neonate.

\section{Patients and methods}

\section{Study design}

This study was carried out between February 1, 1995 and January 31, 1996 and involved all Italian infants born in 65 hospitals located in 17 Italian regions. Owing to the
*Division of Neonatology, Careggi University Hospital of Florence, Florence, and **Laboratory of Epidemiology and Biostatistics, Istituto Superiore di Sanità, Rome, Italy.

Correspondence: C. Dani

Division of Neonatology

Careggi University Hospital

University of Florence School of Medicine

Viale Morgagni, 85

Firenze

Italy

Fax: 39055412900

Keywords: Respiratory distress syndrome risk factors

transient tachypnoea

Received: July 141998

Accepted after revision January 281999 fact that almost all infants in Italy are born in hospitals and that every unit in this study covered a geographically defined area, the newborn infants in a total and unselected population could be studied.

The participating hospitals were classified, according to international practice [4], as level III hospitals with full resources for obstetric and neonatal intensive care $(n=35)$ or county level II hospitals with full resources for neonatal intensive care (these hospitals seldom refer mothers with high-risk pregnancies or neonates with surgical illnesses or other particular pathologies to level III hospitals $(\mathrm{n}=30))$. The incidence of RDS, TT, meconium aspiration syndrome (MAS), persistent foetal circulation (PFC) and pneumonia were evaluated. Furthermore, the incidence of the main associated complications, such as bronchopulmonary dysplasia, intraventricular haemorrhage, necrotizing enterocolitis, patent ductus arteriosus, and air leak were analysed. For all newborn infants, form No. 1 was filled in. The data requested included birth date, sex, singleton or twin, gestational age (calculated on the basis of chronological criteria), birthweight, Apgar score, congenital malformations, type of delivery, induction of foetal lung maturity (steroids or ambroxol) and disorders of pregnancy. For the infants with respiratory disorders, form No. 2 was also filled in; it included the diagnosis of respiratory disease, the type of respiratory support (oxygen therapy, continuous positive airway pressure, mechanical ventilation and surfactant treatment), the occurrence of major complications, maternal age, pregnancy number, the type of delivery and whether or not induction of foetal 
lung maturity with corticosteroids had been performed. In addition, form 2 included disorders of pregnancy such as gestosis, placenta praevia or placental abruption, intrauterine growth retardation, maternal fever (of $>24 \mathrm{~h}$ duration), premature rupture of foetal membranes (PROM), gestational and preconceptional diabetes and isoimmunization. In the event that the infant was transferred to another hospital, form 2 was completed by the physician in charge of the patient at that hospital.

All infants born in participating hospitals were enrolled in the study; to exclude the loss of data in all participating hospitals, the number of infants born was compared to the number of infants enrolled in the study.

Induction of lung maturity was considered to have been performed in cases in which a full course of betamethasone (two doses of $12 \mathrm{mg} \cdot 24 \mathrm{~h}^{-1}$ ) was administered to the mother.

Risk factors were evaluated only in newborn infants affected by RDS or TT, due to the small number of patients affected by the other respiratory diseases studied (MAS, PFC, pneumonia). The following were considered possible risk factors: 1) maternal parity; 2) type of delivery (vaginal delivery, operative vaginal delivery with forceps or vacuum, elective caesarean section (CS) or emergency CS); 3) birthplace in Northern, Central or Southern Italy; 4) single or multiple pregnancy; 5) presence of one or more maternal diseases; 6) female or male sex; 7) birthweight; 8) gestational age; and 9) maternal age.

The Apgar score was not considered a risk factor for RDS and TT, due to its poor value in predicting the development of both acute respiratory disorders and perinatal asphyxia in newborn infants with gestational ages $<30$ weeks and birthweights $<1,500 \mathrm{~g}[5,6]$.

Among risk factors, perinatal asphyxia was not considered due to the lack of the necessary information in the collected data (i.e. signs and symptoms of neurological sequelae and multiorgan system dysfunction, and blood gas analysis showing metabolic or mixed acidaemia) for making a firm diagnosis [5].

\section{Diagnostic criteria}

Respiratory distress syndrome. The criteria for RDS include: oxygen-dependence increasing during the first 24 $\mathrm{h}$; absence of any sign of infection; and a typical radiological pattern with reduced air content, reticulogranular pattern of the lung, and air bronchograms.

Transient tachypnoea. The criteria for TT include: any oxygen supplement requirement during the first $6 \mathrm{~h}$ which does not increase during the subsequent $18 \mathrm{~h}$; the usual improvement in clinical condition within 3-6 h; and chest radiographs which are either normal or show reduced translucency, infiltrates and hyperinsufflation of the lungs.

All the infants affected by respiratory failure who had: 1) a suggestive maternal history (PROM, fever during the $12 \mathrm{~h}$ antepartum or amnionitis); 2) clinical signs of infection (temperature instability, hypotension, metabolic acidosis, poor feeding, diminished activity or lethargy); 3 ) significant results from laboratory studies (anomalies of white blood cell count and of immature-to-total-neutrophil (I/T) ratio, increase in C-reactive protein levels or positive blood and/or bronchotracheal sample cultures); and 4) a radiographic pattern compatible with lung infection, were considered affected by pneumonia and excluded from the group of infants affected by RDS or TT.

\section{Statistical methods}

Wald's test and the Chi-squared test were utilized to evaluate the statistical significance of qualitative and quantitative variables. Logistic regression analysis was used to assess the independent relationship between the onset of RDS or TT and risk factors. A p-value $<0.05$ was considered statistically significant. All variables were included in a stepwise multivariate logistic regression analysis using a backward elimination procedure. At each stage the variable with the largest p-value was eliminated, and the procedure started again, leading to the elimination of another variable (again, the one with the greatest p-value), until all of the remaining variables were statistically significant $(\mathrm{p}<0.05)$.

To simplify interpretation of the results, odds ratios (ORs) and their $95 \%$ confidence intervals (CIs) were calculated by means of multivariate logistic regression analysis, using a low-risk reference group: 1) first pregnancy; 2) vaginal delivery; 3) single pregnancy; 4) absence of maternal disease; 5) female sex; 6) birthweight $\geq 2,500 \mathrm{~g}$; 7) gestational age $>36$ weeks; 8 ) maternal age $<32$ yrs, to evaluate the effect of each of the eight risk factors considered.

\section{Results}

Of 63,537 live infants enrolled in this study, 2.2\% $(1,427)$ developed an acute respiratory disorder. The clinical and demographic data of infants affected by RDS and TT are reported in table 1 .

The incidence of RDS was $1.16 \%(n=734)$, with an overall mortality of $24 \%(176 / 734)$. Mortality was inversely related to gestational age (table 2 ). The antenatal induction of lung maturity with steroidal maternal treatment was performed in $74.9 \%(550 / 734)$ of affected infants. The Apgar score at the 1st minute was $<4$ in $32.3 \%$ (237/ $734)$ of affected newborns, $4-6$ in $37.2 \%(273 / 734)$ and $>7$ in $30.5 \%(224 / 734)$. The Apgar score at the 5th min was $<4$ in $5.4 \%(40 / 734)$ of affected newborns, $4-6$ in $31.3 \%(230 / 734)$ and $>7$ in $63.2 \%(464 / 734)$.

RDS was more frequent at the first or after the fourth pregnancy, after emergency CS, when the mother was $>32$ yrs old, when the mother was affected by a disease of pregnancy, when the infant was male or a twin, and when the birthweight and gestational age were $<1,500 \mathrm{~g}$ or $<36$ weeks, respectively.

Wald's test and the Chi-squared test showed that: 1) gestational age, 2) type of delivery, 3) birthweight, 4) sex, and 5) maternal age, were risk factors for RDS (table 3 ). Multivariate regression analysis confirmed the role of gestational age, birthweight and maternal age as risk factors for RDS and, moreover, demonstrated that elective and emergency CS, among different types of delivery, were risk factors, as was male sex in comparison with female sex. These results were confirmed by calculation of ORs and their 95\% CIs (table 4).

The incidence of TT was $0.93 \%(n=594)$, with an overall mortality of $1.3 \%(8 / 594)$ (table 2$)$. The Apgar score at the 1 st minute was $<4$ in $8.1 \%(48 / 594)$ of affected newborns, 
Table 1. - Clinical and demographic data of newborn infants affected by respiratory distress syndrome (RDS) and transient tachypnoea (TT)

\begin{tabular}{|c|c|c|c|}
\hline & Total n & RDS n (\%) & TT n (\%) \\
\hline $\mathrm{n}$ & & 734 & 594 \\
\hline \multicolumn{4}{|l|}{ Birthweight } \\
\hline$<1500$ & 1036 & $404(39.0)$ & $77(7.4)$ \\
\hline $1500-2499 \mathrm{~g}$ & 3864 & $257(6.7)$ & $223(5.8)$ \\
\hline$>2500 \mathrm{~g}$ & 58224 & $73(0.1)$ & $294(0.5)$ \\
\hline \multicolumn{4}{|l|}{ Gestational age } \\
\hline$<36$ weeks & 3407 & $681(20.0)$ & $350(1.0)$ \\
\hline $\begin{array}{l}36-42 \text { weeks } \\
>42 \text { weeks }\end{array}$ & 59990 & $53(0.1)$ & $244(0.4)$ \\
\hline \multicolumn{4}{|l|}{ Maternal age } \\
\hline$<32$ yrs & 40152 & $442(1.1)$ & $377(0.9)$ \\
\hline$\geq 32$ yrs & 22556 & $292(1.3)$ & $217(1.0)$ \\
\hline \multicolumn{4}{|l|}{ Number of pregnancy } \\
\hline First & 28066 & $336(1.2)$ & $282(1.0)$ \\
\hline Second & 20335 & $191(1.0)$ & $181(0.9)$ \\
\hline Third & 7230 & $90(1.2)$ & $59(0.8)$ \\
\hline Fourth & 2395 & $30(1.3)$ & $31(1.3)$ \\
\hline Fifth or more & 1351 & $25(1.9)$ & $23(1.7)$ \\
\hline \multicolumn{4}{|l|}{ Delivery type } \\
\hline Vaginal & 43941 & $215(0.5)$ & $226(0.5)$ \\
\hline Operative vaginal & 1621 & $1(0.1)$ & $13(0.8)$ \\
\hline Elective CS & 11021 & $158(1.4)$ & 157 (1.4) \\
\hline Emergency CS & 1351 & $360(26.6)$ & $198(14.7)$ \\
\hline \multicolumn{4}{|l|}{ Sex } \\
\hline Male & 32806 & $412(1.3)$ & $342(1.0)$ \\
\hline Female & 30703 & $322(1.1)$ & $252(0.8)$ \\
\hline Twins & 1531 & $167(10.9)$ & $94(6.1)$ \\
\hline Singletons & 62006 & $567(0.9)$ & $500(0.8)$ \\
\hline \multicolumn{4}{|l|}{ Maternal diseases } \\
\hline Yes & 62708 & $475(0.8)$ & $269(0.4)$ \\
\hline No & 62708 & $259(0.4)$ & $325(0.5)$ \\
\hline
\end{tabular}

CS: Caesarean section.

$4-6$ in $28.8 \%(171 / 594)$ and $>7$ in $63.1 \%(375 / 594)$. The Apgar score at the 5 th minute was $<4$ in $1.3 \%(8 / 594)$ of affected newborns, $4-6$ in $8.6 \%(51 / 594)$ and $>7$ in $90.1 \%$ $(535 / 594)$.

The antenatal induction of lung maturity with steroidal maternal treatment was performed in $72.4 \%$ (430/594) of affected infants. This respiratory disorder resulted more

Table 2. - Mortality of newborn infants with respiratory distress syndrome (RDS) and transient tachypnoea (TT) in relation to gestational age

\begin{tabular}{ccc}
\hline Weeks & RDS & TT \\
\hline$<24$ & $10 / 11(90.9)$ & 0 \\
24 & $25 / 30(83.3)$ & 0 \\
25 & $18 / 25(72)$ & $0 / 1(0)$ \\
26 & $19 / 36(52.8)$ & $0 / 1(0)$ \\
27 & $22 / 47(46.9)$ & $1 / 5(20)$ \\
28 & $23 / 58(39.6)$ & $0 / 4(0)$ \\
29 & $15 / 70(21.4)$ & $3 / 11(27.2)$ \\
30 & $15 / 78(19.2)$ & $0 / 15(0)$ \\
31 & $9 / 86(10.5)$ & $0 / 25(0)$ \\
32 & $5 / 88(5.7)$ & $2 / 41(4.9)$ \\
33 & $1 / 52(1.9)$ & $0 / 44(0)$ \\
34 & $3 / 41(7.3)$ & $0 / 76(0)$ \\
35 & $2 / 39(5.1)$ & $0 / 65(0)$ \\
36 & $1 / 20(5)$ & $0 / 62(0)$ \\
$>36$ & $8 / 53(15)$ & $2 / 244(0.8)$ \\
Total & $176 / 734(24)$ & $8 / 594(1.3)$ \\
\hline Data &
\end{tabular}

Data are presented as ratio $(\%)$. frequently after the third pregnancy, after CS, when the infant was male or a twin, and when birthweight and gestational age were $<1,500 \mathrm{~g}$ or $<36$ weeks, respectively.

Wald's test and the Chi-squared test showed that: 1) gestational age, 2) type of delivery, 3) maternal diseases, 4) twin pregnancy, 5) birthweight, and 6) sex were risk factors for TT (table 5). Multivariate regression analysis confirmed the role of gestational age, maternal diseases, twinning and birthweight on the onset of TT and, moreover, demonstrated that operative vaginal delivery, elective and emergency CS and male sex were risk factors. These results were confirmed by calculation of ORs and their $95 \%$ CI (table 6).

\section{Discussion}

Knowledge of epidemiological data permits assessment of possible changes in the incidence of pathologies and their risk factors. Analysis of epidemiological data cannot

Table 3. - Chi-squared and p-values of the statistically significant risk factors for respiratory distress syndrome

\begin{tabular}{lrr}
\hline Variable & Chi-squared & p-value \\
\hline Gestational age & 3241.677 & $<0.001$ \\
Delivery type & 187.588 & $<0.001$ \\
Birthweight & 125.089 & $<0.001$ \\
Sex & 6.579 & 0.010 \\
Maternal age & 3.892 & 0.049 \\
\hline
\end{tabular}


Table 4. - Coefficients of multiple correlation (R), p-values, odds ratios (ORs) and 95\% confidence intervals (Cls) of the statistically significant risk factors for respiratory distress syndrome

\begin{tabular}{|c|c|c|c|c|}
\hline Variable & $\mathrm{R}$ & p-value & OR & $95 \% \mathrm{CI}$ \\
\hline Elective CS versus vaginal delivery & 0.0511 & $<0.0001$ & 1.88 & $1.42-2.48$ \\
\hline Emergency CS versus vaginal delivery & 0.1176 & $<0.0001$ & 3.46 & $2.69-4.44$ \\
\hline Male versus female & 0.0258 & 0.011 & 1.30 & $1.06-1.60$ \\
\hline Birthweight & -0.1272 & $<0.0001$ & 0.88 & $0.86-0.90$ \\
\hline Gestational age & -0.1706 & $<0.0001$ & 0.72 & $0.69-0.75$ \\
\hline Maternal age & -0.0167 & 0.0488 & 0.98 & $0.96-0.99$ \\
\hline
\end{tabular}

CS: Caesarean section.

exclude comparison with previous data and, with regard to this aspect, some observations should be made: 1) in recent years, few studies regarding risk factors for RDS and TT have been performed and it is, therefore, difficult to compare the present results with those of other reports; 2) owing to the introduction in the 1990s of induction of lung maturity and other advances in neonatal respiratory support (e.g.: surfactant and high-frequency oscillatory ventilation), the findings of certain papers, such as the Swedish study of Hualmarson [1], can no longer be utilized; 3) many epidemiological studies can only partially be compared to the present survey because they investigated newborn infants selected by disorders and birth weight, and did not study general newborn population as in the present case.

The current prospective survey confirmed that RDS and TT are frequent neonatal acute respiratory disorders and that the mortality rates caused by them are higher in newborn infants with lower gestational age. The occurrence of antenatal prophylaxis for RDS $(44.9 \%)$ and TT $(72.4 \%)$ by maternal treatment with steroids was very high in this population. The newborns studied by LENOIR et al. [6] received antenatal steroidal treatment in $5.5 \%$ of cases; these data are not reported by MONSET-COUCHARD et al. [7] and are not comparable to those of the Padua study group (14\% in infants born before 34 weeks' gestation) [2]. The high frequency of neonatal respiratory disease prevention could partially explain the reduction in RDS incidence that was observed in comparison with previous studies performed in Sweden (1.15\% versus $2.9 \%)$ [1] and in Italy $(3.3 \%)[2]$. When considering the occurrence of TT, an incidence similar to that previously observed in Italy $(0.93$ versus $1.12 \%)$ was found, however further comparative analysis could not be performed due do to the lack of other comparable data in the literature.

As stated two decades ago in previous studies, the present work confirmed that the frequency of RDS is inversely related to gestational age and birthweight $[1,8,9]$, that CS can favour the onset of RDS $[2,6,7,10]$ and that male sex is a strong risk factor $[1,2,9,11]$ for $\mathrm{RDS}$.

Table 5. - Chi-squared and p-values of the statistically significant risk factors for transient tachypnoea

\begin{tabular}{lcc}
\hline Variable & Chi-squared & p-value \\
\hline Gestational age & 850.719 & $<0.001$ \\
Delivery type & 156.357 & $<0.001$ \\
Maternal diseases & 33.579 & $<0.001$ \\
Twinning & 18.051 & $<0.001$ \\
Birthweight & 14.578 & $<0.001$ \\
Sex & 13.626 & $<0.001$ \\
\hline
\end{tabular}

Logistic regression analysis did not demonstrate relations between maternal diseases and RDS, probably due to the fact that infants of mothers with maternal diseases are commonly subjected to predisposing insults, such as premature birth and CS, which represent the real risk factors [8]. In confirmation of this interpretation, HubBell et al. [12] in a series of almost 500 consecutive infants of diabetic mothers, could not demonstrate any role for maternal diabetes as an independent variable in RDS because of the high CS rate. Moreover UsHER et al. [13] in another study, concluded that the observed association of maternal diabetes and RDS is attributable to the obstetric practice of premature delivery by CS rather than to diabetes per se.

It was observed that advanced maternal age is a risk factor for RDS, a correlation that has never been found before; such a finding could possibly be explained by the increased levels of maternal diseases (hypertension, diabetes, placenta praevia and placental abruption) in older women, conditions which are related to more frequent preterm deliveries [14] and CS, as reported above.

Transient tachypnoea of the newborn is usually a benign self-limiting disease [15]. However, affected infants may experience morbidity due to hypoxaemia [16], pulmonary air leaks [17] and persistent pulmonary hypertension [16, $18,19]$. The most commonly proposed mechanism is a delay in reabsorption of lung fluid [16], but recently a role for a slight immaturity of the surfactant system has been suggested [20].

The present data are in partial agreement with previous reports [16-19, 21, 22], confirming that the onset of TT is favoured by prematurity [16], and that it is more frequent in infants born by CS $[17,21,22]$, those whose mothers were affected by maternal diseases [17], male infants $[17,19,21,22]$, and twins, the latter never having been previously reported in the literature.

It was observed that TT, although commonly considered a typical respiratory disease of near-term or term infants, not infrequently affected very low birthweight infants $(7.4 \%)$ with a significant case fatality rate $(7.8 \%)$. Furthermore, in disagreement with RAwLINGS and SMITH [22], none of the affected newborns had a birthweight $>4,000$ $\mathrm{g}$, excluding the possibility that macrosomia favours the development of TT.

In conclusion, the present data confirm the role of gestational age, birthweight, caesarean section delivery and male sex as risk factors for respiratory distress syndrome and transient tachypnoea; demonstrate that advanced maternal age is a risk factor for respiratory distress syndrome; and rule out the possibility that maternal diseases can act as independent risk factors for respiratory distress syndrome. 
Table 6. - Coefficients of multiple correlation (R), p-values, odds ratios (ORs) and 95\% confidence intervals (Cls) of the statistically significant risks factors for transient tachypnoea

\begin{tabular}{lrrr}
\hline Variable & $\mathrm{R}$ & $\mathrm{p}$-value & OR \\
\hline Operative versus non-operative vaginal delivery & 0.0278 & 0.0092 & 2.12 \\
Elective CS versus vaginal delivery & 0.0658 & $<0.0001$ & 1.86 \\
Emergency CS versus vaginal delivery & 0.1080 & $<0.0001$ & 2.86 \\
Male versus female & 0.0430 & 0.0002 & $1.48-2.33$ \\
Twinning & 0.0416 & 0.0004 & $2.25-3.63$ \\
Maternal diseases & 0.0669 & $<0.0001$ & $1.16-1.67$ \\
Birthweight & -0.0497 & $<0.0001$ & 1.81 \\
Gestational age & -0.1441 & $<0.0001$ & 0.96 \\
\hline
\end{tabular}

CS: Caesarean section.

This study represents a recent reference for the evaluation of maternal and perinatal risk factors for the development of respiratory distress syndrome and transient tachypnoea.

Members of the Italian Group of Neonatal Pneumology. Project Leader: F.F. Rubaltelli (Firenze). Central Staff: C. Dani (Firenze), L. Bonafé (Padova), A. Spagnolo, M. Tangucci (Roma). Northern Italy: U. De Vonderweid (Trieste), F. Macagno (Udine), O.S. Saia (Treviso), E.M. Padovani (Verona), G.F. Temporin (Rovigo), R. Malossi (Trento), L. Gentili (Bolzano), L. Magni, C. Colnaghi (Rho), P. Tagliabue (Monza), A.M. Tempra (Bergamo), A. Cavazza (Brescia), E. Polito (Pavia), C. Fabris (Torino), A. Costantini (Alessandria), W Bonacci, G. Serra (Genova), F. De Maria, L. Lucchi (Bologna), P. Garani, P. Guerrini (Ferrara), C. Boni, C. Magnani (Reggio Emilia), S. Costa Zaccarelli (Modena). Central Italy: A. Boldrini (Pisa), R. Bracci (Siena), S. Tofani, P.L. Duvin (Scandicci), G. Caramia (Ancona), M.S. Lungarotti (Perugia), S. Placidi, G.F. Spennati (L'Aquila), G. Licata, G. Marzetti, P. Danhaive (Rome). Southern Italy: F. Cicale, C. D'Ascoli, P. Giliberti, A. Pugliese, R. Saggiani, A. Scarcella, V. Tripodi (Napoli), G. Citro (Salerno), G. Chirichiello (Avellino), A Mautone (Bari), S. Mastropasqua (Acquaviva), G. Rinaldi (Foggia), M. Petracca (Lecce), P. Cristalli, (San Giovanni Rotondo), A. Mari, G.F. Scarpelli (Cosenza), U. Di Mita, S.M. Vitaliti, R. Pomò (Palermo), M.I. Bonanno Conti, C. Borruso (Trapani), F. Chiappe, D. Rosatelli (Cagliari), C. Corchia (Sassari)

\section{References}

1. Hjalmarson O. Epidemiology and classification of acute neonatal respiratory disorders. A prospective study. Acta Paediatr Scand 1981: 70: 773-783.

2. Bonafè L, Rubaltelli FF. The incidence of acute neonatal respiratory disorders in Padova county: an epidemiological survey. Acta Paediatr 1996; 85: 1236-1240.

3. Rubaltelli FF, Dani C, Reali MF, et al. Acute neonatal respiratory distress in Italy. A one year prospective study. Acta Paediatr 1998; 87: 1261-1268.

4. James LS. Perinatal events and respiratory distress syndrome. N Engl J Med 1975; 132: 1291-1292.

5. Carter BS, Haverkamp AD, Merenstein GB. The definition of acute perinatal asphyxia. Clin Perinatol 1993; 20 : 287-304.

6. Lenoir M, Grandjean H, Leloup M, Claris O, Hascoet JM. Devenir a court et moyen term d'une cohorte de 1157 nouveau-nes atteints de detresse respiratoire. Arch Pediatr 1994; 1: 1004-1010.

7. Monset-Couchard M, de Bethmann O, Kastler B. Midand long-term outcome of 89 premature infants weighing less than $1000 \mathrm{~g}$ at birth, all appropriate for gestational age. Biol Neonate 1996; 70: 328-338.

8. Farrell PM, Avery ME. Hyaline membrane disease. Am Rev Respir Dis 1975; 111: 657-685.

9. Fedrick J, Butler NR. Hyaline membrane disease. Lancet 1972; 2: 768-769.

10. Finnstrom O, Otterbladd Olausson P, Sedin G, et al. The Swedish national prospective study on extremely low birthweight (ELBW) infants. Incidence, mortality, morbidity and survival in relation to level of care. Acta Paediatr 1997; 86: 503-511.

11. Torday JS, Nielsen HC. The sex difference in foetal lung surfactant production. Exp Lung Res 1987; 12: 1-19.

12. Hubbell JP, Drorbaugh JE, Muirhead DM. The newborn infant of diabetic mother. Med Clin North Am 1965; 49: 1035-1046.

13. Usher RH, Allen AC, McLean FH. Risk of respiratory distress syndrome related to gestational age, route of delivery, and maternal diabetes. Am J Obstet Gynecol 1971; 111: 826-839.

14. Fretts RC, Schmittdiel J, McLean FH, Usher RH, Goldman MB. Increased maternal age and the risk of fetal death. $N$ Engl J Med 1995; 333: 953-957.

15. Avery ME, Gatewood OB, Brumley G. Transient tachypnea of newborn. Am J Dis Child 1966; 111: 380-385.

16. Bucciarelli RL, Egan EA, Gessner IH, Eitzman DV. Persistence of fetal cardiopulmonary circulation: one manifestation of transient tachypnea of the newborn. Pediatrics 1976; 58: 192-197.

17. Gross TL, Sokol RJ, Kwong MS, Wilson M, Kunhert PM. Transient tachypnea of the newborn: the relationship to preterm delivery and significant neonatal morbidity. Am J Obstet Gynecol 1983; 146: 236-241.

18. Tuddehope DI, Smyth MH. Is "transient tachypnea of the newborn" always a benign disease? Report of six babies requiring mechanical ventilation. Aust Paediatr J 1979; 15: $160-165$.

19. Halliday HL, McLure G, McReid M. Transient tachypnea of the newborn: two distinct clinical entities? Arch Dis Child 1981; 56: 322-325.

20. Song GW, Sun B, Curstedt T, Grossman G, Robertson B. Effect of amyloride and surfactant on lung liquid clearance in mechanically ventilated newborn rabbits. Respir Physiol 1992; 88: 233-246.

21. Wiswell MT, Rawlings JS, Smith FR, Goo ED. Effect of furosemide on the clinical course of transient tachypnea of the newborn. Pediatrics 1985; 75: 908-910.

22. Rawlings JS, Smith FR. Transient tachypnea of the newborn. An analysis of neonatal and obstetric risk factors. Am J Dis Child 1984; 138: 869-871. 